\title{
Electronic transport in penta-graphene nanoribbon devices using carbon nanotube electrodes: A computational study
}

\author{
M. Shunaid Parvaiz ${ }^{1,2}$, Khurshed A. Shah ${ }^{1, *}$, G. N. Dar ${ }^{2}$, Sugata Chowdhury ${ }^{3}$, Olasunbo Farinre ${ }^{3}$ Prabhakar Misra $^{3}$ \\ ${ }^{1}$ Department of Physics, S. P. College Campus, Cluster University, Srinagar, J\&K-190001, India \\ ${ }^{2}$ Department of Physics, University of Kashmir, Srinagar, J\&K-190006, India \\ ${ }^{3}$ Department of Physics and Astronomy, Howard University, Washington, DC 20059, USA \\ *drkhursheda@gmail.com
}

DOI 10.17586/2220-8054-2020-11-2-176-182

\begin{abstract}
Electronic transport properties of pristine, homogenously and heterogeneously boron-nitrogen doped saw-tooth penta-graphene nanoribbon (SPGNR) with carbon nanotube electrodes have been studied using Extended Huckel Theory in combination with the non-equilibrium Green's function formalism. CNT electrodes produce a remarkable increase in current at higher bias voltages in pristine SPGNR. The current intensity is maximum at higher bias voltages, while the nitrogen-doped model shows current from the onset of the bias voltage. However, there are also considerable differences in the I-V curves associated with the pristine model and other models doped homogenously as well as heterogeneously with boron and nitrogen. The doped models also exhibit a small negative differential resistance effect, with much prominence in the nitrogen-doped model. In summary, our findings show clearly that doping can effectively modulate the electronic and the transport properties of penta-graphene nanoribbons that have not been studied and reported thus far.
\end{abstract}

Keywords: Penta-graphene nanoribbon, CNT, NEGF, EHT, Doping, ATK.

Received: 10 March 2020

Revised: 23 March 2020

\section{Introduction}

Carbon is one of the most interesting elements, having a number of allotropes like graphite, diamond, fullerene, nanotube, $\mathrm{C}_{60}$ and graphene [1]. After the successful synthesis of graphene, significant research has been conducted in characterizing carbon-based nanomaterials [2-5]. A large number of 2D carbon allotropes are being studied. Although some of the polymorphs, such as graphdiyne, are metastable compared with graphene, they have been successfully synthesized [6]. Some of the 2D allotropes of carbon are being researched for their interesting properties that are an improvement on graphene, such as anisotropic Dirac cones, ferromagnetic nature, high catalytic behavior and high superconductivity, due to the high density of states at the Fermi level [7-9]. These results reveal that the novel properties of carbon allotropes are related to the topological arrangement of carbon atoms and thus highlight the importance of structure-property relationships [10].

Carbon nanostructures are based on two important structural motifs, namely pentagons and hexagons. The hexagon is the building block of zero-dimensional nanoflakes, nanotube, graphene, graphite and metallic carbon phases [11-13]. Carbon materials composed of pentagons are rarely found. Carbon pentagons are generally known as topological defects, or geometrical frustrations, as stated in the well-known "isolated pentagon rule" (IPR) for fullerenes, where pentagons must be separated from each other by neighboring hexagons to reduce the steric stress $[14,15]$. For example, $\mathrm{C}_{60}$ is made up of 12 pentagons separated by 20 hexagons resembling the shape of a soccer ball, explaining the IPR rule perfectly. In some examples, the existence of carbon pentagons is also accompanied by carbon heptagons, which are separated from one another [14]. The synthesis of pentagon-based $\mathrm{C}_{20}$ cage has inspired many researchers and considerable research has been done to stabilize pentagon-based and non-IPR carbon materials of different dimensionalities [16]. Some non-IPR fullerenes have also been experimentally synthesized [15]. During the growth of 2D carbon sheets, a "pentagon first" mechanism was brought into play in order to transform sp carbon chains to $\mathrm{sp}^{2}$ carbon rings [17]. Therefore, we wanted to pursue the idea of building a penta-graphene carbon sheet using fused pentagons as structural motivation. The dynamical, thermal, and mechanical stability of such a structure has been confirmed in a series of publications [18-24]. Moreover, Zhang and coworkers [12] have reported the thermal and mechanical stability of pentagon-based carbon nanotubes (CNTs). Additionally, Avramov et al. [25] have reported the binding energy per atom for $(n, n)$ penta-graphene based carbon nanotubes (CNTs), confirming that the $(7,7)$ penta-graphene based CNT is the most energetically stable structure. Pentagraphene is an attractive material for optoelectronic applications, because it is a semiconductor with a quasi-direct band gap. Additionally, it has a reduced thermal conductivity as compared to graphene and is an auxetic material which means it has a negative Poisson's ratio. 
Furthermore, many properties of pentagraphene can be tuned by doping and functionalization, therefore it will be an exciting material in the coming times.

In the present work, we investigate the electrical property of a new carbon allotrope, penta-graphene, consisting entirely of pentagons, within the framework of Extended Huckel theory (EHT). As far as we are aware, we show for the first time that the penta-graphene nanoribbon contacted with CNT electrodes exhibit higher current and conductance values as compared to similar pristine pentagraphene nanoribbon (PGNR) with PGNR electrodes as reported by Tien, et. al. [26]. Also, its boron-nitrogen doped versions sandwiched between two carbon nanotube electrodes exhibit interesting electronic properties that are different than those reported in previous studies cited earlier. The current paper is organized from here on as follows: Section II describes the models and methods employed in the study, Section III presents the results and discussion, and concluding remarks are presented in Section IV.

\section{Models and methods}

In the present study, we have modeled a saw-tooth penta-graphene nanoribbon (SPGNR) based on a two-probe system attached with two (4,0) zig-zag CNT electrodes using the Atomistic Toolkit (ATK) (version P-2019.03) software and its graphical interface virtual nanolab [27], as shown in Fig. 1. CNT electrodes have been used in previous research work designed to study the electronic properties of various devices [28].

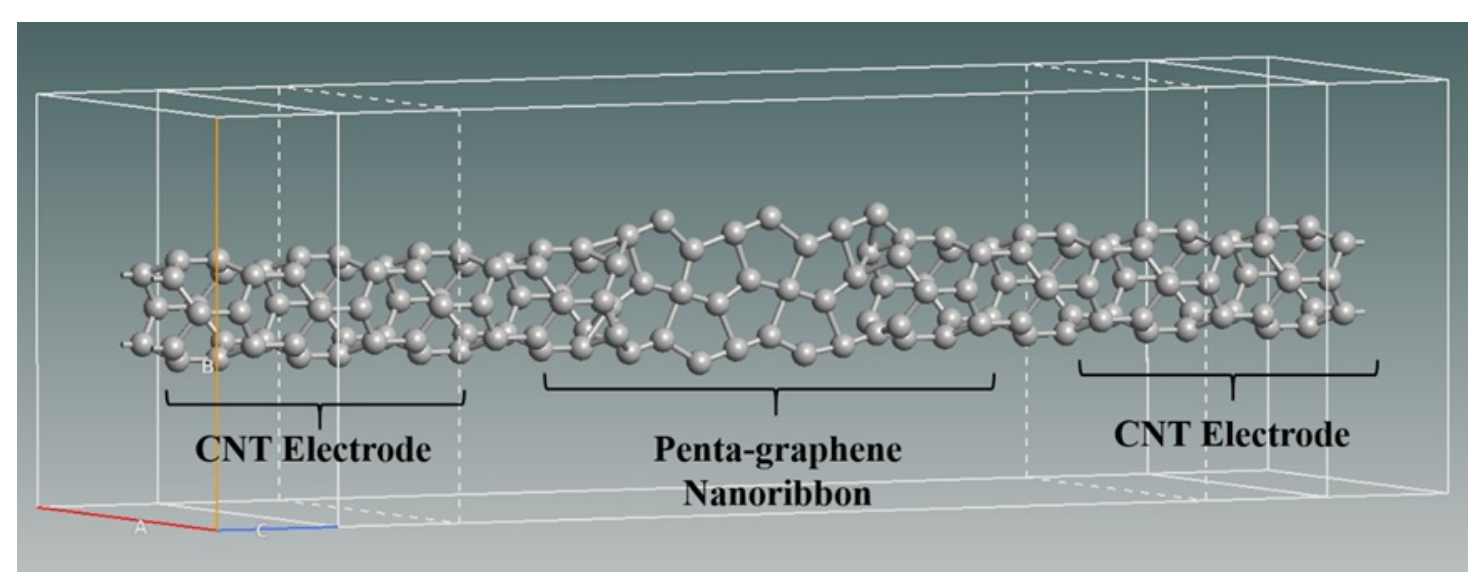

FIG. 1. The computational model of SPGNR with CNT electrodes

In order to understand the behavior of the PGNR-based system for comparative studies, we substitutionally doped the scattering region of the device with 4 atoms of boron and nitrogen, both homogenously and heterogeneously, as illustrated in Fig. 2(a,b,c). In all simulated models, both electrodes consist of 16 atoms each, and the central scattering region consists of 120 atoms. The central scattering region was taken as $30 \AA$ and the length of the two electrodes was set at $4.263 \AA$ each. To overcome the scattering losses, $10 \%$ of the length of electrodes was considered as the scattering region.

Here, we have based our hypothesis on the Non-Equilibrium Green's Function (NEGF) formalism and used the single particle approach based on the Landauer-Buttiker formalism to determine the current [29]

$$
I=\frac{2 e}{h} \int_{-\infty}^{+\infty} T\left(E, V_{b}\right)\left[f\left(E-\mu_{L}\right)-f\left(E-\mu_{R}\right)\right] d E
$$

where $T\left(E, V_{b}\right)$ is the transmission function at energy $E$ and voltage $V_{b}$, and $f\left(E-\mu_{L}\right)$ and $f\left(E-\mu_{R}\right)$ are the Fermi distribution functions of the left and right electrodes, respectively.

The models used in the study were simulated using Huckel parameters with an electrode temperature of $300 \mathrm{~K}$. The Density mesh cut-off was set to 55 Hartee. The set of k-points were chosen as 1, 1, 125 for appropriate sampling. The maximum range for the interaction was taken as $10 \AA$ and the Fourier2D solver was adopted as the Poisson solver for the boundary conditions. The applied bias across the two electrodes was varied from 0 to $2 \mathrm{~V}$ in order to analyze the current and the transmission spectra. Table 1 summarizes the calculation parameters used for the simulation associated with the model. 


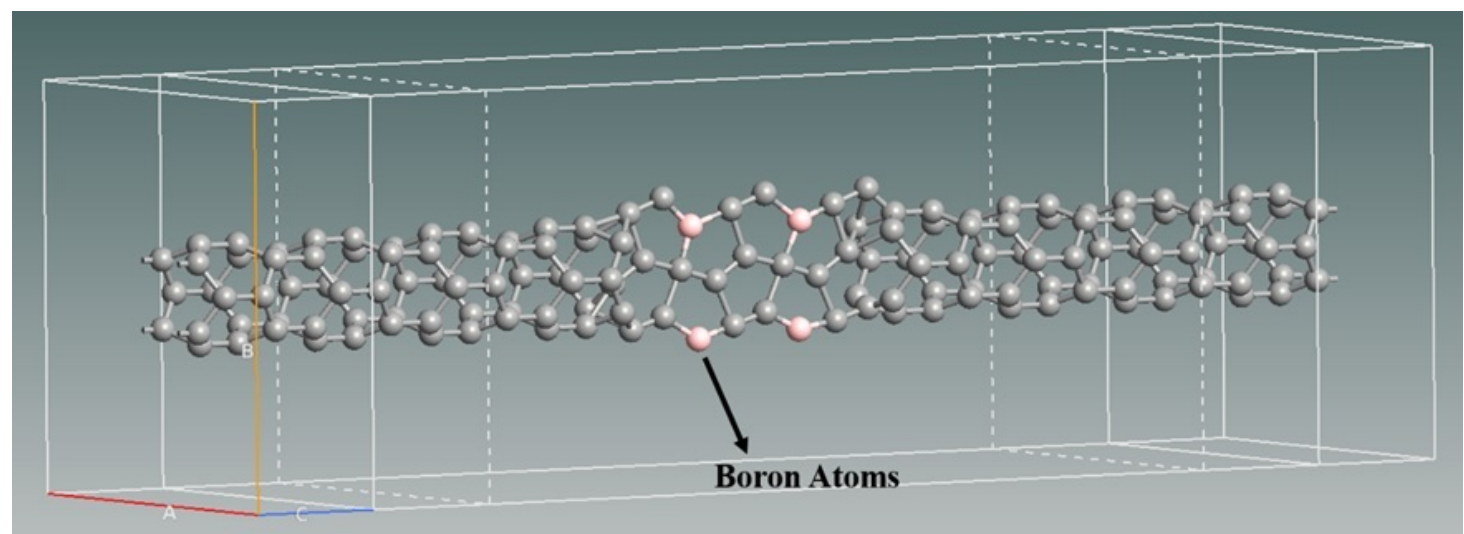

(a)

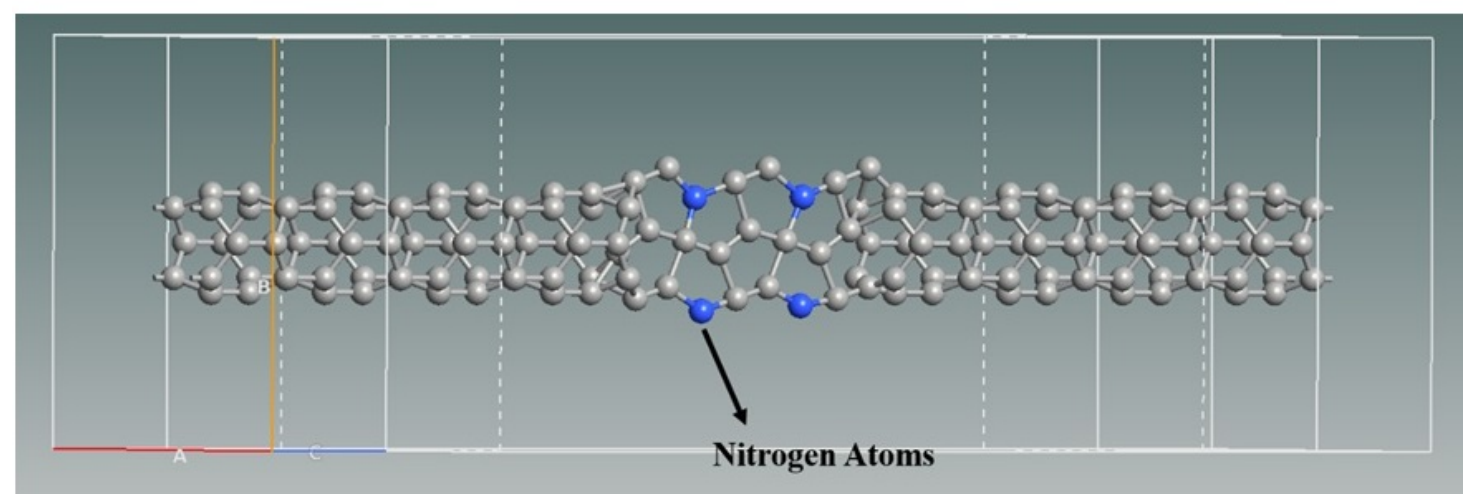

(b)

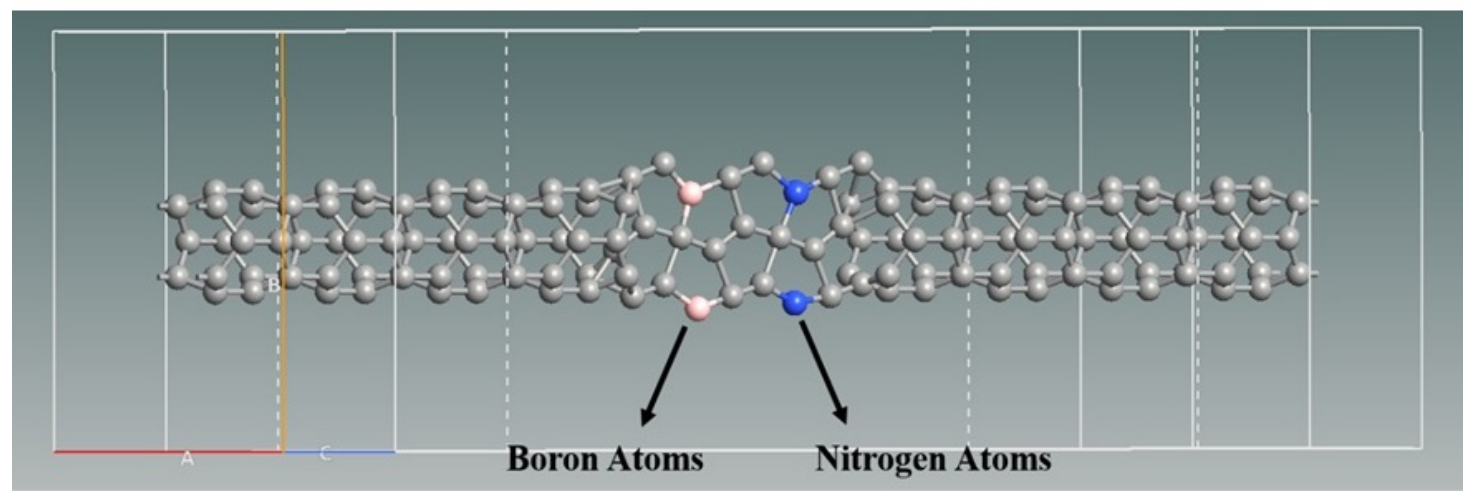

(c)

FIG. 2. PGNR models doped with (a) boron atoms, (b) nitrogen atoms, and (c) both boron and nitrogen atoms 
TABLE 1. Simulation parameters associated with the model

\begin{tabular}{|c|c|}
\hline Parameter & Value \\
\hline \hline Calculator & Semi Empirical \\
\hline Formalism & Extended Huckel Theory (EHT) \\
\hline Density mesh cut-off & $1 \times 1 \times 125$ \\
\hline k-point sampling & 100 \\
\hline Maximum Steps & $0.01 \mathrm{~nm}$ \\
\hline Step Size & Fourier2D solver \\
\hline Poisson Solver & $300 \mathrm{~K}$ \\
\hline Electrode Temperature & Non-equilibrium Green's Function (NEGF) \\
\hline Device Algorithm Formalism
\end{tabular}

\section{Simulation results}

All the proposed models were simulated in device mode using the ATK software and its graphical interface, Virtual Nanolab, in order to study the transport properties and the effect of different doping atoms on the conductance of the CNT contacted PGNR two-probe system. All of the structures were fully relaxed in order to make the residual forces on each atom smaller than $0.05 \mathrm{eV} / \AA$. In order to achieve the requisite results, we analyzed the projected device density of states (PDDoS) and transmission spectra of all models and plotted the I-V curves and conductance curves under different bias voltages. Fig. 3 shows the projected PDDoS of the proposed models. In case of pristine SPGNR, the states of $\mathrm{C}$ atoms are almost non-existent in the energy zone, with a similar trend in all models doped with $\mathrm{B}$. In the case of N-SPGNR, new electronic states of $\mathrm{N}$ atoms are observed in the energy zone corresponding to the band gap of the pristine SPGNR, and thereby reducing the band gap. This in turn also results in the change of the band structure leading to a high current in N-SPGNR at low applied voltages. It is observed that the alteration in band structure is mainly dependent upon the doped element and not on the electronic distribution from the dopants [30].

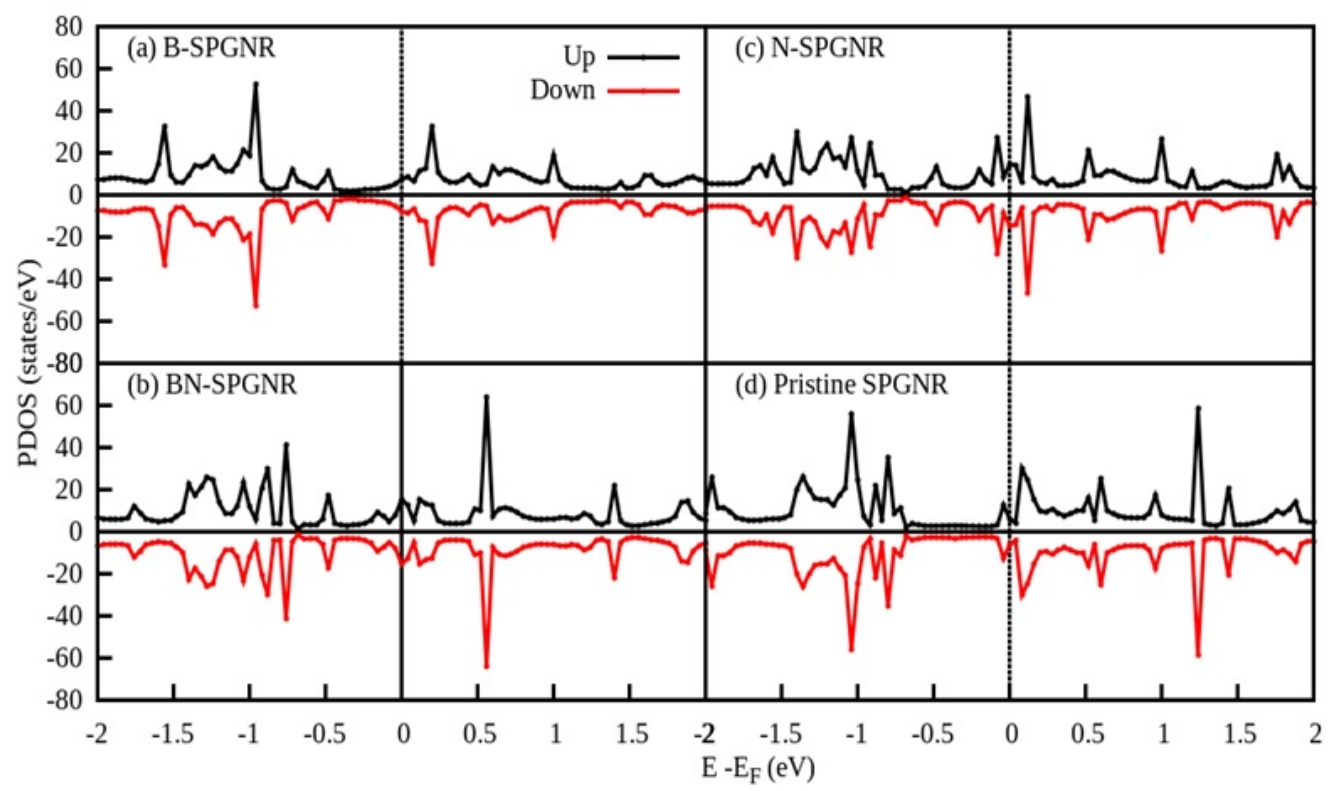

FIG. 3. Projected Device Density of States (PDDoS) for (a) B-SPGNR (b) BN-SPGNR (c) NSPGNR, and (d) Pristine SPGNR

Figure 4(a) shows the I-V curves of pristine, boron-doped, nitrogen-doped and heterogeneously boron-nitrogen doped systems, respectively. The results show that the pristine SPGNR model produces the maximum current at higher applied voltages, while the nitrogen- doped model produces a high current at lower applied voltages. Using CNT electrodes results in a remarkable increase in current at higher bias voltages in pristine SPGNR, as compared to other reports [26]. The possible mechanism for this behavior is due to the fact that the CNT exhibits a ballistic 
transport property and acts as an efficient charge injecting electrode in this model due to negligible contact resistance associated with the C-C bonds of the CNT electrodes and the SPGNR scattering region. At higher applied voltages, the charge transfer from the CNT electrodes is so high that a bandgap reduction takes place in the SPGNR, resulting in high output current. For pristine, boron-doped and heterogeneously boron-nitrogen doped models, the current changes slightly as the bias voltage increases from $0 \mathrm{~V}$ to $0.6 \mathrm{~V}$. The current behavior of the pristine penta-graphene system starts to increase at a bias voltage of $0.8 \mathrm{~V}$, reaches its maximum at $1.6 \mathrm{~V}$ and then decreases afterwards. In the boron-doped model, the first peak appears at $1.0 \mathrm{~V}$, followed by a sharp increase in current reaching its maximum value at $1.6 \mathrm{~V}$ and then decreasing to its minimum at $2.0 \mathrm{~V}$. A similar trend is observed in the BN-doped model, where the current starts to increase at $0.8 \mathrm{~V}$, forming one peak at $1.4 \mathrm{~V}$ and then reaching up to the highest value at $1.8 \mathrm{~V}$. However, the current intensity in these three models is high with peak intensity of $13.774 \mu \mathrm{A}$ in the pristine model. On the other hand, the current intensity of the nitrogen-doped model increases linearly with the bias voltage from $0 \mathrm{~V}$ to $0.8 \mathrm{~V}$; the current then decreases at $1.0 \mathrm{~V}$ and subsequently increases again at $1.2 \mathrm{~V}$ and remains almost constant with the rest of the bias voltage. This variation of current with the bias voltage is due to the difference in electronic scattering of different dopant atoms. Nitrogen has five outer electrons, which means that it will have free electrons in the doped penta-graphene nanoribbon configuration; this in turn results in a high electron density, thereby explaining the high value of current at low bias.

a

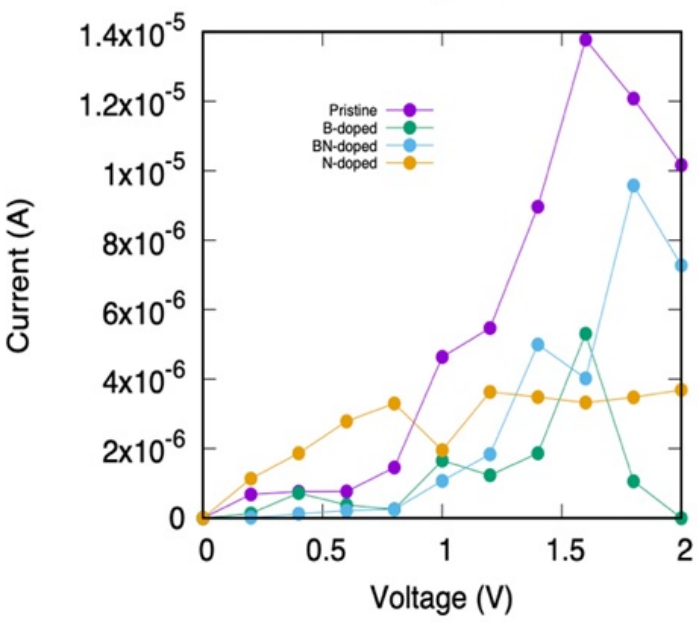

b

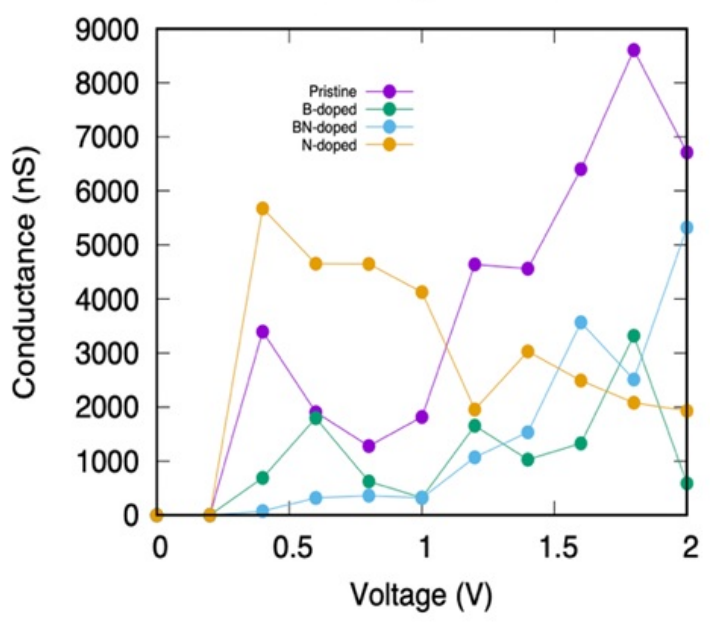

FIG. 4. (a) I-V curves of Pristine SPGNR, B-SPGNR, BN-SPGNR and N-SPGNR (b) Conductance curves of Pristine SPGNR, B-SPGNR, BN-SPGNR and N-SPGNR

Figure 4(b) gives a comparison between the conductance of the proposed models, showing that the nitrogen-doped model has a higher conductance at low voltages while the pristine model shows increased conductance at higher bias voltages. It is important to mention here that penta-graphene is an indirect band-gap semiconductor with a band gap of $3.25 \mathrm{eV}$ and the electronic states near the Fermi level originate from the $\mathrm{sp}^{2}$ hybridized carbon atoms. The $\mathrm{sp}^{3}$ hybridized carbon atoms spatially separate the $\mathrm{p}_{z}$ orbitals of the $\mathrm{sp}^{2}$ hybridized carbon atoms, which screens the full electron delocalization leading to a finite bandgap. The non-dispersive partially degenerate valence bands give high density of states near the Fermi level, thereby leading to high conductance.

In order to further understand the obtained I-V curves of the studied systems, we examined the transmission spectra of the modelled systems at a bias voltage of $0.8 \mathrm{~V}$, as shown in Fig. 5. $T(E)$ almost remains unchanged with the increasing bias voltage to $0.8 \mathrm{~V}$. Nevertheless, there exist higher coefficients at the bias windows from $1.0 \mathrm{~V}$ that leads to the current fluctuation. The outcomes show a strong influence of the transmitted spectrum on the I-V characteristics of the two-probe system. In the nitrogen-doped model, it is observed that the transmittance peaks are present in all the displayed voltages, as compared to other models, showing a strong impact of the transmission spectrum on the I-V characteristics of the two-probe model.

Furthermore, our results show a small negative differential resistance (NDR) behavior in the doped penta-graphene models. The NDR effect is most prominent in the nitrogen doped model. As the voltage is applied, the current arises due to the electrons in the pertinent states and the transmission coefficient in the bias range. The transmission occurs due to the electronic band-to -band tunneling, which is possible only when the bands are shifted in the order of the semiconductor energy gap. In the present investigation, we are using the semiconducting penta-graphene nanoribbon 

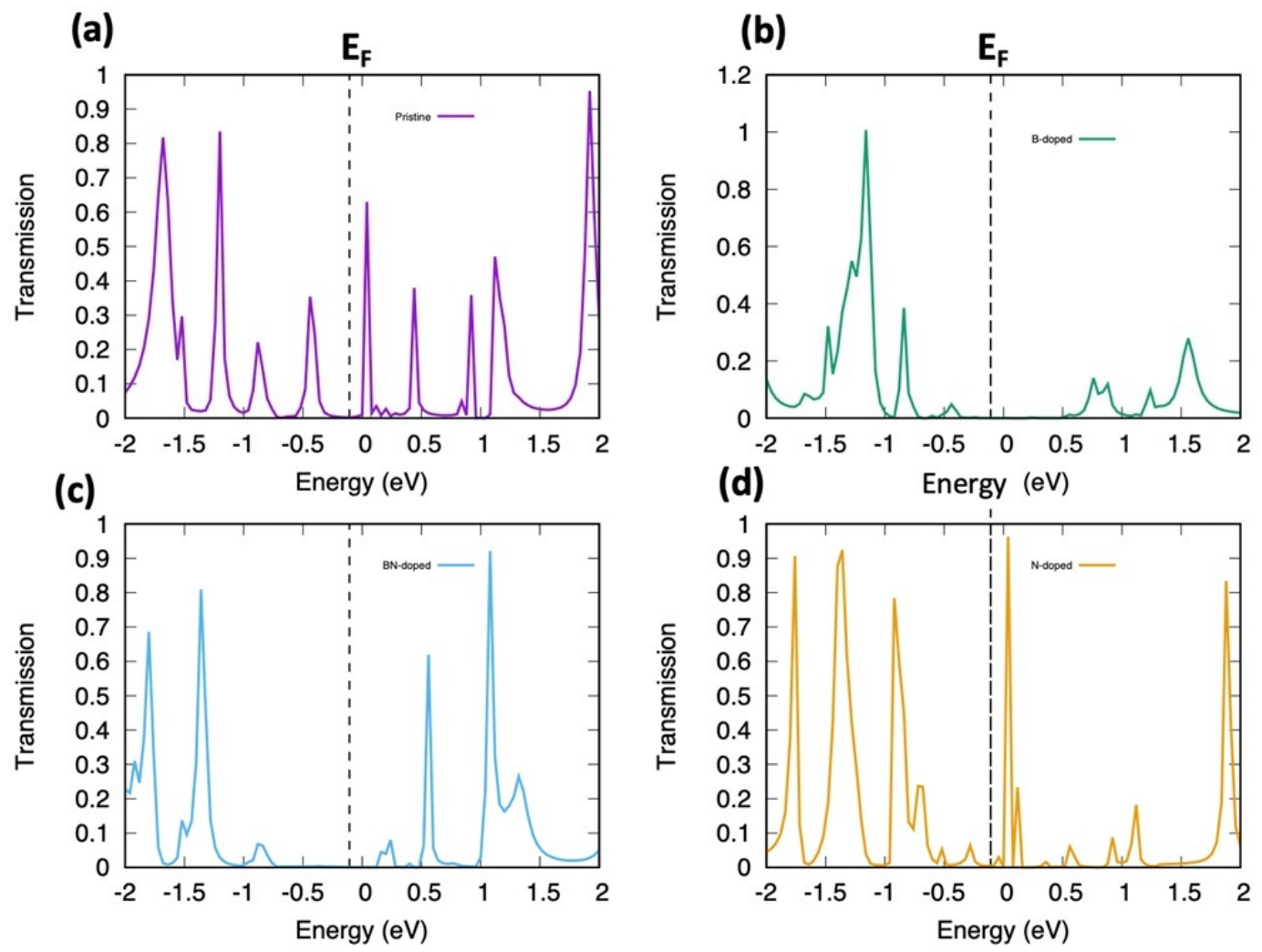

FIG. 5. Transmission spectra of (a) pristine SPGNR, (b) B-doped SPGNR, (c) BN-doped SPGNR and (d) N-doped SPGNR at a bias voltage of $0.8 \mathrm{~V}$

and the nitrogen doping makes band-to-band tunneling feasible by appropriately shifting and aligning of the bands with respect to the Fermi level, and thereby giving rise to the NDR region.

\section{Conclusion}

In conclusion, by analyzing the electronic and transport characteristics of pristine and doped penta-graphene nanoribbons with CNT electrodes, we have observed that using CNT electrodes results in a remarkable increase in current at higher bias voltages in pristine SPGNR as compared to other cited reports. Doping also significantly affects the I-V characteristics and transmission spectra of the two-probe systems. Specifically, the operating voltage of the nitrogen-doped model is significantly reduced as compared to the pristine, homogenously boron-doped and heterogeneously boron-nitrogen doped models. Moreover, the results show that the magnitude of the current in the pristine penta-graphene model is maximum at higher bias voltages. The behavior of the computed transmission spectrum completely matches the I-V characteristics. The small but perceptible NDR behavior is also observed in doped penta-graphene models, especially in the nitrogen-doped model.

\section{Acknowledgement}

This work is supported by the Department of Science and Technology, Science and Engineering Research Board (DST-SERB), New Delhi, India, funded project (Grant No. EMR/002866/2017).

\section{References}

[1] Kroto H.W., Heath J.R., OBrien, S.C., Curl R.F., Smalley R.E. C60: Buckminsterfullerene. Nature, 1985, 318, P. 162-165.

[2] Shah K.A., Parvaiz M.S. Computational comparative study of substitutional, endo and exo BN Co-Doped single walled carbon nanotube system. Superlattices and Microstructures, 2016, 93, P. 234-241.

[3] Shah K.A., Parvaiz M.S. Negative differential resistance in BN co-doped coaxial carbon nanotube field effect transistor. Superlattices and Microstructures, 2016, 100, P. 375-380.

[4] Shah K.A., Parvaiz M.S., Dar G.N. Photocurrent in single walled carbon nanotubes. Physics Letters A, 2019, 383, P. $2207-2212$.

[5] Zwanenburg F.A., Van der Mast D.W., et. al. Electric Field Control of Magnetoresistance in InP Nanowires with ferromagnetic Contacts. Nano Letters, 2009, 9, P. 2704-2709. 
[6] Li Y., Xu L., Liu H. Chem, Graphdiyne and graphyne: from theoretical predictions to practical construction. Chem. Soc. Rev., 2014, 43, P. 2572-2586.

[7] Malko D., Neiss C., Vines F., Gorling A. Competition for Graphene: Graphynes with Direction-Dependent Dirac Cones. Phys. Rev. Lett., 2012, 108, P. 086804.

[8] Mina M., Susumu O. Two-Dimensional $\mathrm{sp}^{2}$ Carbon Network of Fused Pentagons: All Carbon Ferromagnetic Sheet. Appl. Phys. Express, 2013, 6, P. 095101.

[9] Terrones H., et al. New Metallic Allotropes of Planar and Tubular Carbon. Phys. Rev. Lett., 2000, 84, P. 1716-1719.

[10] Xu L.C., et al. Two dimensional Dirac carbon allotropes from graphene. Nanoscale, 2014, 6, P. 1113-1118.

[11] Bucknum M.J., Hoffmann R. A Hypothetical Dense 3,4-Connected Carbon Net and Related $\mathrm{B}_{2} \mathrm{C}$ and $\mathrm{CN}_{2}$ Nets Built from 1,4Cyclohexadienoid Units. J. Am. Chem. Soc., 1994, 116, P. 11456-11464.

[12] Zhang S., Wang Q., Chen X., Jena P. Stable three-dimensional metallic carbon with interlocking hexagons. Proc. Natl. Acad. Sci., 2013, 110, P. 18809-18813.

[13] Omachi H., Nakayama T., Takahashi E., Segawa Y., Itami K. A grossly warped nanographene and the consequences of multiple oddmembered-ring defects. Nat. Chem., 2013, 5, P. 739-744.

[14] Deza M., Fowler P.W., Shtogrin M., Vietze K. Pentaheptite Modifications of the Graphite Sheet. J. Chem. Inf. Comput. Sci., 2000, 40, P. 13251332.

[15] Tan Y.Z., Xie S.Y., Huang R.B., Zheng L.S. The stabilization of fused-pentagon fullerene molecules. Nat. Chem., 2009, 1, P. 450-460.

[16] Prinzbach H., et al. Gas-phase production and photoelectron spectroscopy of the smallest fullerene, C20. Nature, $2000,407, \mathrm{P} .60-63$.

[17] Wang Y., et al. Template Effect in the Competition between Haeckelite and Graphene Growth on Ni(111): Quantum Chemical Molecular Dynamics Simulations. J. Am. Chem. Soc., 2011, 133, P. 18837-18842.

[18] Yagmurcukardes M., Sahin H., Kang J., Torun E., Peeters F.M., Senger R.T. Pentagonal monolayer crystals of carbon, boron nitride, and silver azide. J. Appl. Phys., 2015, 118, P. 104303-104306.

[19] Yu Z.G., Zhang Y.W. A comparative density functional study on electrical properties of layered penta-graphene. J. Appl. Phys., 2015, 118, P. 165706.

[20] Xu W., Zhang G., Li B. Thermal conductivity of penta-graphene from molecular dynamics study. J. Chem. Phys., 2015, 143, P. 154703154706.

[21] Cranford S.W. When is 6 less than 5? Penta-to hexa-graphene transition. Carbon, 2016, 96, P. 421-428.

[22] Lopez-Bezanilla A., Littlewood P.B. $\sigma-\pi$-Band Inversion in a Novel Two-Dimensional Material. J. Phys. Chem. C, 2015, 119, P. 1946919474.

[23] Stauber T., Beltran J.I., Schliemann J. Tight-binding approach to penta-graphene. Sci. Rep., 2016, 6, P. $22672-22680$.

[24] Rajbanshi B., Sarkar S., Mandal B., Sarkar P. Energetic and electronic structure of pentagraphene nanoribbons. Carbon, 2016, 100, P. 118-125.

[25] Avramov P., Demin V., Luo M., Choi C.H., Sorokin P.B., Yakobson B., Chernozatonskii L. Translation Symmetry Breakdown in LowDimensional Lattices of Pentagonal Rings. J. Phys. Chem. Lett., 2015, 6, P. 4525-4531.

[26] Tien N.T., Thao P.T.B., Phuc V.T., Ahuja R. Electronic and transport features of sawtooth penta-graphene nanoribbons via substitutional doping, Physica E: Low-dimensional Systems and Nanostructure, 2019, 114, P. 113572.

[27] Quantum ATK version P-2019.03, Synopsis Quantum ATK (www.synopsys.com/silicon/quantumatk.html).

[28] Baughman R.H., Zakhidov A.A., de Heer W.A. Carbon nanotubes-the route toward applications. Science, 2002, 297, P. 787-792.

[29] Landauer R. Spatial Variation of Currents and Fields Due to Localized Scatterers in Metallic Conduction. IBM J. Res. Dev., 1957, 1, P. 223231.

[30] Berdiyorov G., Dixit G., Madjet M. Band gap engineering in penta-graphene by substitutional doping: first-principles calculations, J. Phys.: Condensed Matter, 2016, 28, P. 475001-475010. 